\title{
The Mereological Structure of Informational Entities
}

\author{
Adrien BARTON ${ }^{\mathrm{a}, \mathrm{b}, 1}$, Fumiaki TOYOSHIMA ${ }^{\mathrm{b}}$, Laure VIEU ${ }^{\mathrm{a}, \mathrm{c}}$, \\ Paul FABRY ${ }^{\mathrm{b}}$ and Jean-François ETHIER ${ }^{\mathrm{b}, 2}$ \\ anstitut de Recherche en Informatique de Toulouse, CNRS, France \\ ${ }^{\mathrm{b}}$ GRIIS, Université de Sherbrooke, Quebec, Canada \\ ${ }^{c}$ Laboratory for Applied Ontology, ISTC-CNR, Trento, Italy
}

\begin{abstract}
This article provides the basis of a formal axiomatic system for a mereology of informational entities based on the idea of information fillers that can occupy information slots, such as the same word that can be used in different sentences. It is inspired by Karen Bennett's mereological system that enables a whole to have a part "twice over", but differs from it in several key points, such as the acceptance of empty slots, and the possibility for slots to have slots. Information slots are analyzed as informational entities that can carry aboutness.
\end{abstract}

Keywords. Mereology, Information content entity, Informational structure

\section{Introduction}

Documents are a primary source of data. Consider the field of medicine: many data about which medications a patient takes (or is likely to take) are extracted from prescription documents written to him or her by doctors, or from drug dispensing reports written by pharmacists [1]. Forms and surveys are another important source of data.

As we will see below, documents receive growing attention in information systems and ontologies. However, the informational entities that compose such documents are often characterized simply as entities that are "about" something, and their analysis is too basic to enable an accurate and manageable representation of data and information in various fields. To address this problem, we will provide a mereological analysis of the structure of informational entities, as the meaning of a complex informational entity depends on the meanings of its parts (although maybe not only on those). Interestingly enough, this task will lead to a foundational challenge to reconsider classical mereological systems that are traditionally used in formal ontology. We will here only consider documents (and more generally informational entities) that are composed in natural language, excluding pictures, musical partitions, etc. - although this limitation could be lifted.

\footnotetext{
${ }^{1}$ Corresponding Author: Adrien Barton, IRIT, 118 Route de Narbonne, F-31062 Toulouse Cedex 9, France; E-mail: adrien.barton@irit.fr.

2 Corresponding Author: Jean-François Ethier, Université de Sherbrooke, 2500 Boulevard de l'Université, Sherbrooke, QC, J1K 2R1, Canada; E-mail: ethierj@gmail.com.
} 


\section{Preliminaries and state-of-the-art works}

\subsection{Mereology among universals or particulars}

Mereology is a formal study of the part-whole relation, which constitutes a mainstay of ontological practice. We may consider building a mereology at two levels: between particulars, or between universals. This article will focus on a mereology of particulars of informational entities. Former work by Masolo and Vieu [2] has focused on a mereology of universals (not necessarily those of informational entities). Particulars of informational entities and universals of non-informational entities share some commonalities [3, p. 105-107], such as the ability to be multiply localized. This is reflected in the fact that Bennett's [4] mereological work, used for building a mereology of universals in the work by Masolo and Vieu [2], will also serve as a basis for our formal ontology of informational entity particulars.

\subsection{Classical extensional mereology}

Different mereological systems have been proposed. The most standard is sometimes informally called "Classical Extensional Mereology" (CEM) (not necessarily in Simon's [5] sense). CEM embraces "ground mereology" [6] according to which parthood is a (partial) ordering relation (reflexive, antisymmetric, and transitive) and that accepts the two following principles (the former being entailed by the latter):

- Weak Supplementation Principle (WSP): If $\mathrm{x}$ is a proper part of $\mathrm{y}$, then there is some $\mathrm{z}$ such that $\mathrm{z}$ is a part of $\mathrm{y}$ and $\mathrm{z}$ is disjoint from (i.e. does not overlap) $\mathrm{x}$.

- Strong Supplementation Principle (SSP): If $y$ is not part of $x$, then there is some $\mathrm{z}$ such that $\mathrm{z}$ is a part of $\mathrm{y}$ and $\mathrm{z}$ is disjoint from $\mathrm{x}$.

Note that SSP implies that two different entities cannot have exactly the same proper parts. For instance, the upper ontology DOLCE [7] builds upon "general extensional mereology" [6] satisfying both WSP and SSP; whereas the upper ontology Basic Formal Ontology (BFO 2.0) [3] adopts a "minimal extensional mereology" [5] including WSP but not SSP. Although Simons [5] endorses WSP and recommends dropping out SSP, WSP is still controversial [8] (for an overview of the decidability of various mereological theories, see [9]).

Most importantly, CEM is committed to the principle that an entity $\mathrm{x}$ cannot have an entity $y$ as a part many times over [4][10]. We will reject this principle below because it turns out to be unsuitable for developing a mereological account of informational entities.

\subsection{Informational entities in conceptual modeling and ontology}

There are some existing works on informational entities in the domain of conceptual modeling and ontology. For instance, the Functional Requirements for Authority Data (FRAD) [11] introduces an entity named "expression": "The intellectual or artistic realization of a work in the form of alphanumeric, musical, or choreographic notation, sound, image, object, movement, etc., or any combination of such forms." To take another example, the Unified Nations System Document Ontology (UNDO) [12] aims to provide a framework for the formal description of all entities and the relations that 
hold among them in the documents of the United Nations. Both the FRAD and the UNDO are largely practically motivated and they leave room for meticulous ontological analysis of documents and information. Finally, CIDOC [13] is a lightweight ontology aimed at cultural heritage domain. It includes a mereological relation for information objects ("incorporates"), but its axiomatization is very limited.

Two ontologies based on BFO deal with informational entities. First, the Information Artifact Ontology (IAO) [14] introduces the class Information Content Entity (ICE), the instances of which can be documents, databases, and digital images, as a subclass of Generically Dependent Continuant (whose instances, intuitively, "can migrate from one bearer to another through a process of copying" [6] [p. 179]). ICEs are about some "portion of reality", a label which encompasses all the BFO particular entities (including other ICEs) but also universals, relations, and "configurations" (e.g., the cat being on the mat). Second, the Information Entity Ontology in the Common Core Ontologies (CCO) [15] identifies three subkinds of the IAO:is about relation, namely: "describes" (used for e.g., reports and representations), "prescribes" (used for e.g., plans and artifact specifications), and "designates" (used for e.g., names and other identifiers).

Garbacz [16] has proposed an ontology of texts, that include considerations on parthood, precedence, identity and location. Masolo et al. [17] introduced a notion of description based on DOLCE, characterized as follows: "different expressions (...) can be associated to the same description" (generic dependence) and "descriptions must be encoded on (...) physical supports" (concretization). This notion has been exploited within ontology patterns for describing information objects [18], but a full-fledged formal ontology of informational entities has not been pursued within DOLCE yet. Finally, the formal system we will present has some analogies with feature structures see 5.7 for a short discussion.

\section{A classification of informational entities}

\subsection{A brief consideration on informational entities, aboutness, and semiotics}

Documents have a dual face: physical objects on one hand (e.g. a copy of the book Labyrinths), and informational content that can be concretized by such physical objects on the other hand (e.g. the informational content shared by all such copies of Labyrinths). We will here be interested in this second sense of documents.

Many documents are constituted by sentences, which can be decomposed in words. Words written in alphabetic systems can be decomposed in letters. All those entities will be called here "Informational entities" (IEs). The models of informational entities presented above seem to share the premise that informational entities are about (synonyms: "refers to", "represents", "mentions") something. This is indeed often the case with documents, (declarative) sentences therein, and words that constitute the sentences: e.g., the word "cat" can be generally taken to be about the class Cat. It may not be the case of all informational entities, however. For instance, it is debatable whether letters such as "A" are about anything (although one might argue that they are about sounds). Therefore, IEs should not be identified with ICEs from IAO, as they are not necessarily about something.

Aboutness remains a notoriously elusive notion, despite some recent philosophical works [19]. We will not delve into its nature (but see section 5.4), as some groundwork on mereology is necessary to adequately deal with this issue, but will presuppose that 
aboutness emerges from a semiotic system. Relatively neglected in ontology [20], semiotics analyzes representations in terms of the triad of a sign, an object and an interpreter. That is to say, meaning is "an attribution of significance by some sign users for other sign users for some designated purposes" [20] [p. 120]. Therefore, for example, the letter " $A$ " might be about something, but only when somebody refers to " $A$ " with the intention of conveying some meaning (e.g., an excellent grade) to another person. To take seriously semiotic considerations in ontology may require a foundational investigation into language [20], which is outside the scope of this paper. What we will seek below is a mereological theory of informational entities without assuming any reference to an aboutness relation (but see section 5.4).

\subsection{The need for information slots}

It seems that some informational entities can be found in several documents: for example, the IE 'flu' can be found in a part dict 0 in a medical dictionary that reads ' $f l u=$ an infectious disease caused by an influenza virus', and in a (here idealized) diagnosis diag 0 written by Dr. House about John Doe that reads: 'John Doe / flu / Dr. House'. This raises two challenges. First, the same IE could appear several times in the same document. For example, suppose that another line in the same diagnosis document reads 'John Doe / asthma / Dr. Jones': 'John Doe' would then appear twice in the document. Second, two IEs that would play different roles would be instances of different classes that would naturally be seen as disjoint (e.g. 'flu' as an instance of Diagnosed disease specification in a diagnosis document versus 'flu' as an instance of Therapeutic indication in a drug prescription that prescribes to take some medication in case of flu). To account for this, we will introduce, following Bennett [4], the classes of Information Slot (IS) (somewhat akin to CCO's Information Structured Entity, although to our knowledge, the nature of the latter has not been investigated in detail yet) and Information Filler (IF), both being subclasses of the class of IE: the same individual IF 'flu' can be found in both $\operatorname{diag}_{0}$ and dict $_{0}$, as filling different individual ISs (note that such an ontology of slots and fillers fits also especially well with a machine-readable language such as XML).

Similarly, consider the chains of characters $\mathrm{IE}_{1}={ }^{\prime} \mathrm{ab}$ ' and $\mathrm{IE}_{2}=$ ' $\mathrm{ba}$ ' (for theories of strings, see [21,22]): we will consider that IE ${ }_{1}$ has two ISs ' $1{ }^{\text {st }} l e t t e r_{1}[]$ ' and ' $2^{\text {nd }}$ letter 1[] ', and that $\mathrm{IE}_{2}$ has two ISs ' $1{ }^{\text {st }} \operatorname{letter}_{2}[]$ ' and ' $2^{\text {nd }} \operatorname{letter}_{2}[]$ '. The same individual filler ' $a$ ' occupies ' 1 st $\operatorname{letter}_{1}[]$ ' and ' $2^{\text {nd }} \operatorname{letter}_{2}[]$ ', and the same individual filler ' $b$ ' occupies ' $1{ }^{\text {st }}$ letter 2[] ' and ' $2^{\text {nd }}$ letter $[$ []' (although we will not deal here with the representation of order relations between slots).

\subsection{Information slots and information fillers}

Suppose that in a hospital, all diagnostic documents have the following structure: 'patient[] condition[] doctor[]'. That is, any diagnostic report at this hospital has (at least) three ISs, each of which can be filled by an IF. When we write "t $\mathrm{x}]$ ", $\mathrm{x}$ refers to an IF and $t$ to an IS that is filled by $x$.

We will also consider that IEs can have a structure even if this structure is not filled - that is, they can have a "mere mereological structure" (see section 5.6). This means that in our ontology, an IS does not need to be filled by an IF.

Note that two documents of the same type do not have the same ISs, although they can be filled with the same kinds of IFs (or even the same IFs). Suppose that Dr. House fills the document $\operatorname{diag}_{0}$ : 'patient 0 ['John Doe'] condition 0 ['Flu'] doctoro['Dr. House']' 
and $\operatorname{diag}_{1}$ : 'patient ${ }_{1}$ ['Jane Brown'] condition ${ }_{1}$ ['Asthma'] doctor ${ }_{1}\left[{ }^{\prime} D r\right.$. House']'. Although $\operatorname{diag}_{0}$ and $\operatorname{diag}_{1}$ have similar structures, they have different particular ISs: 'patient $t_{0}[]$ ' is different from 'patient 1 []', 'doctor 0 []' is different from 'doctor ${ }_{1}[]$ ', etc. However, 'patient 0 []' and 'patient ${ }_{1}[]$ ' are instances of the same class IS for patient name, 'doctoro[]' and 'doctor 1 []' are instances of the same class IS for doctor name, etc. On top of that, the same particular IF 'Dr. House' fills both particular ISs 'doctoro[]' and 'doctor 1 []' - and we could introduce the class $I F$ for doctor name, of which 'Dr. House' is an instance. Therefore, there are both particulars and classes of ISs and IFs.

Finally, as we have seen earlier, not only words can occupy ISs: the same letter can appear in several words by occupying several different ISs. For example, in the word ' $a a$ ', the same particular IF 'a' occupies the IS ' $1{ }^{\text {st }} \operatorname{letter}_{a a}[]$ ' and the IS ' 2 nd $\operatorname{letter}_{a a}[]$ '.

\subsection{Information slot as a generically dependent continuant that can be concretized}

To analyze ISs and IFs, we anchor them in the IAO ontological framework for ICEs in such a way that informational entities are generically dependent upon their bearers (see [23] for a detailed discussion on generic dependence) and exist by being "concretized" (alternatively, see [24] for an analysis of the general notion of slot in terms of grounding and essence). However, we extend this idea to all informational entities, including those that are not ICEs. The letter "A", for instance (even if it is not about anything, and thus not an ICE), may be concretized as an ink pattern on a paper, or as a pixel pattern on a computer screen.

Suppose there are two concretizations of diag 0 : a first one printed on paper, that is concretized by the ink pattern $\mathrm{p}_{1}$, and a second one on my computer screen, concretized by a pixel pattern $\mathrm{p}_{2}$. Let's call $\mathrm{IF}_{1}=$ 'John Doe', $\mathrm{IF}_{2}=$ 'Flu' and $\mathrm{IF}_{3}=$ 'Dr. House' the three IFs that constitute what we will call the "content" of $\operatorname{diag}_{0} . \mathrm{IF}_{1}, \mathrm{IF}_{2}$ and $\mathrm{IF}_{3}$ are concretized by (parts of) $\mathrm{p}_{1}$, as well as (parts of) $\mathrm{p}_{2}$. In our ontological framework, each IS in $\operatorname{diag}_{0}$ is also concretized (at least) twice, since $\operatorname{diag}_{0}$ is concretized twice (in $\mathrm{p}_{1}$ and $\mathrm{p}_{2}$ ). ISs are in this respect similar to IFs.

A difficulty with ISs lies in pinpointing their concretizations: it might be expected that diagnostic sheets at a hospital should be filled with the name of a doctor at the bottom part of the sheet, without anything indicating the need for such a name on the paper. Even if something indicates it, such as the words 'Doctor name' written on the paper, those words are not an IS, but rather an IF that indicates the (otherwise invisible, but socially determined) existence of an IS. Maybe, if the prescription is concretized on a sheet of paper, such an IS would be concretized by a BFO:Site [3, pp. 112-113]. For example, if $\operatorname{diag}_{0}$ is printed on a paper, the slot 'doctor 0 []' in $\operatorname{diag}_{0}$ is concretized by a site on the printed document that is occupied by the ink pattern on the paper concretizing the IF 'Dr. House'. Another possibility would be that ISs are concretized by some cognitive structure in a collective of agents, reflecting on the social nature of ISs. We will not elaborate further on this question in this paper, and instead focus on the axiomatic mereology of informational entities.

\section{An axiomatization of mereological relations among informational entities}

We will first present an axiomatical mereological system for IEs (4.1-4.3) inspired by Bennett's system [4], provide a simple model in 4.4, and then show in 4.5 how this system differs from Bennett's. 


\subsection{Ground axiomatization}

\subsubsection{Key predicates}

Let us adapt Bennett's mereology, where Sty means that $t$ is an IS (what Bennett called a "slot", while she used the notation "Ps" instead of "S") of $y$, Fxt means that $x$ fills $t$, and Pxy means that $\mathrm{x}$ is a part of $\mathrm{y}$. We introduce as primitive the unary predicate IS = "is a slot", and the binary predicates $\mathrm{F}$ and $\mathrm{S}$, where $\mathrm{S}$ is defined on the domain IS:

\section{(AX0) Only slots are slots of something $\quad$ Stx $\rightarrow$ ISt}

We then define the following predicates IF = "is a filler" and HS = "having a slot":

(DEF0) Have-slot and filler

$$
\begin{aligned}
& \mathrm{HSa}:={ }_{\text {def }} \exists \mathrm{t} \mathrm{Sta} \\
& \mathrm{IFx}:=_{\text {def }} \exists \mathrm{t} \mathrm{Fxt}
\end{aligned}
$$

(the variable symbols " t', "u", "v" will be used for slots, "x", “y”, "z" for fillers, and "a" for entities that can be either slots or fillers)

We will build a mereological theory on the domain of fillers and slots. This means that each non-slot entity under consideration is a filler, and therefore fills some slot: diag, for example, would fill a slot 'diagnosiso[]' that may not be a slot of any filler. This conception fits with the idea that there are no "free-floating" IFs, but that they always appear in a context (represented at least partially by the slot they fill) with some social expectations defined by the semiotic system on which they depend.

$\mathrm{x}$ is a proper part of a filler $\mathrm{y}$ if $\mathrm{x}$ fills a slot of $\mathrm{y}$ :

(DEF1) Proper filler-parthood

PPxy: $==_{\text {def }}$ IFy \& $\exists \mathrm{t}($ Sty $\&$ Fxt $)$

Note that IFy is imposed to make sure that PP holds only between fillers (trivially, PPxy $\rightarrow$ IFx \& IFy), so as to avoid that a filler would be a proper part of a slot.

We then define parthood on the basis of proper parthood in a similar way as in [5], among fillers:

(DEF2) Filler-parthood

$$
\text { Pxy:= }{ }_{\operatorname{def}} \text { PPxy } \vee(\text { IFy \& } \mathrm{x}=\mathrm{y})
$$

\subsubsection{First axioms}

In this system, only slots are filled, and slots cannot fill:
(AX1) Only slots are filled
Fxt $\rightarrow$ ISt
(AX2) Slots cannot fill
Fxt $\rightarrow \neg \mathrm{ISx}$

Moreover, no entity fills any of its slots (intuitively, the slots of an entity can only be filled by something "smaller" than this entity):

\section{(AX3) No improper parthood slots $\quad \neg$ (Stx \& Fxt)}

Also, there is at most one filler for a given slot:
(AX4) Max one occupancy
Fyt \& Fzt $\rightarrow \mathrm{y}=\mathrm{z}$

From AX2 and DEF0, we deduce the trivial theorem that fillers and slots are disjoint: (TH0) Fillers are not slots IFx $\rightarrow \neg$ ISx

From AX1 and DEF0, we deduce that the domain of F is IF, and its range is IS; whereas the domain of S is IS, and its range is HS (by definition of HS):
(TH1) Domain and range of $\mathbf{F}$
Fxt $\rightarrow$ IFx \& ISt
(TH2) Domain and range of $\mathbf{S}$
Stx $\rightarrow$ ISt \& HSx

Like in Bennett's theory, this system does not require that each filler occupies exactly one parthood slot. This is indeed the main motivation of the system, to explain 
how IEs such as the letter 'a' or 'John Doe' can occupy several parthood slots of the same entity. That is, an entity can "have a part twice over", in Bennett's slogan.

However, contrarily to Bennett's system (see section 4.5 for a full comparison), a slot may not be filled; and slots can have slots. Indeed, we want to be able to say that on a document, the slot 'cno[]' (for "complete name") has as slots 'fno[]' (for "first name") and ' $\ln _{0}[]$ '(for "last name"), even if ' $\mathrm{cn}_{0}[]$ ' is not filled. Or to say that a slot 'nominal group $_{0}[]$ ' has a slot 'noun 0[] ', even if unfilled [2]. We even accept in our ontology documents that would be mere slot structures without any IF filling them or any of their slots (for example, my homework is currently only a blank page, but has already a predefined structure and thus some slots - see 5.6).

We accept the axioms stating that $\mathrm{S}$ is a strict order relation:

\section{(AX5) Slot-of irreflexivity \\ (AX6) Slot-of asymmetry \\ (AX7) Slot-of transitivity}

$$
\begin{aligned}
& \neg \mathrm{Stt} \\
& \mathrm{Stu} \rightarrow \neg \mathrm{Sut} \\
& \mathrm{Stu} \& \mathrm{Suv} \rightarrow \mathrm{Stv}
\end{aligned}
$$

\subsubsection{Filling and underfilling}

We will now add the relation of underfilling (noted UF): a filler underfills a slot if it fills a slot of this slot:

\section{(DEF3) Underfilling}

$$
\text { UFxt: }={ }_{\operatorname{def}} \text { ISt } \& \exists \mathrm{u}(\text { Sut } \& \text { Fxu) }
$$

Trivially, something that underfills is a filler:

\section{(TH3) Only fillers underfill}

$$
\mathrm{UFxt} \rightarrow \mathrm{IFx}
$$

On the other hand, an underfiller of a slot $t$ is not necessarily a part (in the sense of $\mathrm{P}$ ) of something that fills $\mathrm{t}$ (since the larger slot $\mathrm{t}$ can remain unfilled). Contrast underfilling with proper parthood, as they look axiomatically very similar: $\mathrm{x}$ underfills $\mathrm{t}$ if $\mathrm{x}$ fills a slot of $\mathrm{t}$ and $t$ is a slot; whereas $\mathrm{x}$ is a proper part of $\mathrm{z}$ if $\mathrm{x}$ fills a slot of $\mathrm{z}$ and $z$ is a filler. We can show that both F and UF are strict orders, but those theorems are vacuously true (since the range and the domain of each of those two relations are disjoint).

\subsubsection{Slot inheritance}

The following axiom will play a pivotal role in our theory (and be discussed extensively in 5.1): if a filler $x$ fills a slot $t$, any slot of $x$ is a slot of $t$, and vice versa:

\section{(AX8) Slots of filler are identical to slots of the filled slot $\quad$ Fxt $\rightarrow($ Sux $\leftrightarrow$ Sut)}

For example, if 'John Doe' fills ' $\mathrm{cn}_{0}[]$ ', and ' $\mathrm{fn}_{\mathrm{JD}}[]$ ' is a slot of 'John Doe', then ' $\mathrm{fn}_{\mathrm{JD}}[]$ ' is also a slot of ' $\mathrm{cn}_{0}[]$ '. Consequently, a slot may have a slot for two different reasons. First, it might be because of its own intrinsic structure, such as ' $\mathrm{cn} 0[$ '] having intrinsically the slots ' $\mathrm{fn}_{0}[]$ ' and ' $\mathrm{n}_{0}[]$ '. Second, it might be because of the structure of its filler, such as ' $\mathrm{fn} 0[\mathrm{]}$ ' being filled by 'Jean-Marc' and therefore having as slots ' $1 \mathrm{hfn}_{\mathrm{JM}}[\mathrm{]}$ ' and '2hfnנм[]' (for respectively the first half of the first name and its second half), filled respectively by 'Jean' and 'Marc' (but it would not have such slots if it was filled by 'John').

Using AX8, we can show the following theorems:

- The slots of a part of an entity are slots of that entity too:

(TH4) Slot of part inheritance

Stx \& Pxy $\rightarrow$ Sty 
Proof: Let's suppose that Stx \& Pxy. Since Pxy, there is a u such that Suy and Fxu. From $\mathrm{AX} 8$ and Fxu, we know that for any v: Svx $\rightarrow$ Svu. Applying this to v=t, from Stx we can deduce Stu. From Stu and Suy, we deduce Sty by AX7 (Slot-of transitivity).

- If $\mathrm{x}$ is a proper part of $\mathrm{y}$, then $\mathrm{y}$ is not a proper part of $\mathrm{x}$ :

(TH5) Proper parthood asymmetry

PPxy $\rightarrow \neg$ PPyx

Proof: Suppose that PPxy and PPyx. Then Pxy and y fills a slot $u$ of x. By TH4 (Slot of part inheritance), $u$ is a slot of $y$. Thus, $y$ fills one of its slots, which is impossible by AX3 (No improper parthood slots).

- If $x$ is a proper part of $y$ that fills $t$, then $x$ underfills $t$ :

(TH6) Proper part of a filler underfills the filler's slot $\quad$ PPxy \& Fyt $\rightarrow$ UFxt

Proof: Suppose that Fyt and PPxy. By PPxy, there is a slot $u$ of y filled by $x$. By AX8 and Fyt, $u$ is also a slot of $t$. Thus, $x$ fills a slot of the slot $t$. That is, $x$ underfills $t$.

- An underfiller of a slot does not fill this slot (and vice-versa):

(TH7) Underfiller of a slot does not fill it $\mathrm{UFxt} \rightarrow \neg \mathrm{Fxt}$

Proof: Suppose that UFxt and Fxt. Since UFxt, $x$ fills a slot $u$ of $t$. Since Fxt, any slot of $t$ is a slot of $x$ by AX8. Therefore, $u$ is a slot of $x$. Thus, $x$ fills one of its slots: absurd by AX3 (No improper parthood slots).

\subsection{5. (Filler-)parthood as a partial order}

From the above, we can deduce easily the following theorems (that follow respectively from DEF2, TH5 Proper parthood asymmetry and TH4 Slot of part inheritance):

(TH8) Parthood Reflexivity

(TH9) Parthood Anti-Symmetry

(TH10) Parthood Transitivity

\author{
$\mathrm{IFx} \rightarrow \mathrm{Pxx}$ \\ Pxy \& Pyx $\rightarrow \mathrm{x}=\mathrm{y}$ \\ Pxy \& Pyz $\rightarrow$ Pxz
}

\subsection{Filling axioms and theorem}

Let's now add two axioms from which we can deduce new filling relations. The first one is a "descending" filling axiom: a slot of a filler is always filled:

\section{(AX9) Slot of a filler is filled \\ IFx \& Stx $\rightarrow \exists y$ Fyt}

We can deduce trivially from this axiom that a slot of a filler is always filled by $a$ proper part of the filler:

(TH11) Slot of a filler is filled by a proper part $\quad$ IFx \& Stx $\rightarrow \exists y$ (Fyt \& PPyx)

For example, the slot ' $\mathrm{nn}_{\mathrm{JD}}[]$ ' of the filler 'John Doe' is filled by 'John'.

The second axiom is an "ascending" filling axiom: if all slots of a slot are filled, then this slot is also filled:

(AX10) All sub-slots filled implies slot filled ISt \& $[\forall \mathrm{u}$ (Sut $\rightarrow \exists x$ Fxu) $] \rightarrow \exists y$ Fyt For example, if the two only slots ' $\mathrm{fn}_{0}[]$ ' and ' $\mathrm{n}_{0}[]$ ' of ' $\mathrm{cn}_{0}[]$ ' are filled, then ' $\mathrm{cn}_{0}[]$ ' is filled (see 5.3 for a brief discussion). 


\subsection{Weak supplementation}

Weak supplementation (and therefore strong supplementation also) clearly does not hold in our ontology. For example, 'a' is a proper part of ' $1{ }^{\text {st }} l_{\text {etter }}$ ['a'] $2^{\text {nd }}$ letter $_{1}$ ['a']', but there is no proper part of the latter that would not overlap ' $a$ '. However, we can accept an axiom akin to weak supplementation inspired by Bennett [4]. It states that if $x$ occupies a slot $t$ of the filler $y$, then $y$ has a slot $u$ that is neither identical to $t$, nor a slot of $\mathrm{x}$ :

\section{(AX11) IF Weak Supplementation $\quad$ IFy \& Sty \& Fxt $\rightarrow \exists u$ [Suy \& $\neg(t=u \vee$ Sux $)$ ]}

We leave adoption of axioms akin to strong supplementation for future work (but see the discussion in 5.1.1 below). Note however an interesting point about extensionality: contrarily to classical mereology, two different fillers can have exactly the same fillers as proper parts, such as ' $1{ }^{\text {st }} \operatorname{letter}_{1}\left[{ }^{\prime} a\right.$ '] $2^{\text {nd }} \operatorname{letter}_{1}\left[{ }^{\prime} b\right.$ ']' and ' $1{ }^{\text {st }} l e t t e r_{2}\left[{ }^{\prime} b\right.$ '] $2^{\text {nd }} l_{\text {etter }}[$ ' $a$ ']'.

\subsection{Consistency of the axioms}

To provide a model, we will represent the structure of an IE by inclusion of rectangles (representing ISs) and ellipses (representing IFs):

- An ellipse $x$ immediately inside a box $t$ represents Fxt.

- A box t inside (not necessarily immediately inside, because of AX7 Slot-of transitivity and AX8) an ellipse or a box z represents Stz.

The following simple model on Fig. 1 (where unique names are used) satisfies all of AX1-AX11, therefore this axiomatic system is consistent:

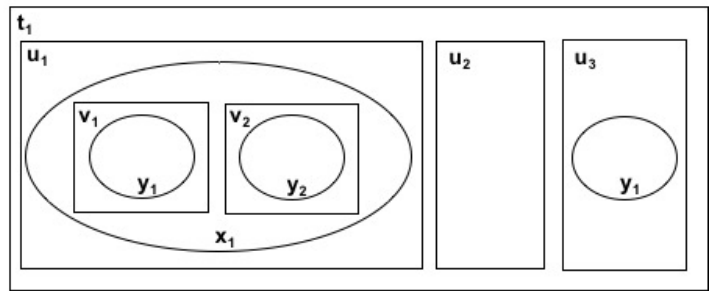

Figure 1. Model of the theory

Future work should investigate further the class of possible models, using a model finder such as Alloy.

\subsection{Comparison with Bennett's mereology}

In the following, for an integer $n$, "A $n$ " or "T $n$ " (e.g. "A3") refers to an axiom or theorem in Bennett's system [4], whereas "AXn" or "TH $n$ " (e.g. "AX3") refers to an axiom or theorem in our theory. There are several important changes in the system presented above, compared to Bennett's mereology. First, not all slots are "slots of something": there can be "free-floating" slots. Second, a filler does not have an "improper slot" that it itself fills (cf. AX3, contra A4): intuitively, a slot is "smaller" than the thing it is a slot of. Third, there can be empty slots, and therefore, contra Bennett's A7 that states that each slot has a single occupant, we merely accept a weaker axiom of maximum one occupant (AX4). Fourth, slots can have slots, contra A3 - and therefore, we introduced the notion of underfilling in DEF3. 
Bennett deduces from her axioms that $\mathrm{S}$ is a partial order, but since slots do not have slots in her theory, those order properties are vacuous. In our system, those order properties are quite substantial (and necessary for many of our demonstrations), and we accepted them as axioms. Bennett also accepts as an axiom the Slot of Part Inheritance (A5). In our case, we accepted the more general axiom AX8 (which would have no sense in Bennett's theory, in which slots do not have slots); we then used it to prove Slot of Part Inheritance as a theorem (TH4).

We accepted a weak supplementation axiom on fillers (AX11) similar to Bennett's theorem T13 (which Bennett calls "Slot Weak Supplementation", whereas we called our axiom "IF Weak Supplementation", for reasons that will become clear in 5.1.1), which she deduces from an axiom of "Slot Strong Supplementation" (A8). Note that the last part of Bennett's T13 axiom reads " $\exists$ u (Suy \& $\neg$ Sux)", whereas it reads for us " $\exists \mathrm{u}$ [Suy $\& \neg(\mathrm{t}=\mathrm{u} \vee$ Sux $)]$ ", where $\mathrm{t}$ is the slot of $\mathrm{y}$ filled by $\mathrm{x}$ mentioned in the left-hand side: indeed, for Bennett, each entity fills one of its slots, but we exclude improper parthood slots, so we need to make sure explicitly that this supplementary slot $u$ is not identical to $\mathrm{t}$.

\section{Discussion}

We will discuss here several ways in which the system proposed above could be developed.

\subsection{The identification of the slots of a filled slot and the slots of its filler}

We have proposed above a relatively simple theory of ISs and IFs, that identifies by AX8 the slots of a filler with the slots of the slot it fills. However, this might raise challenges of two kinds.

\subsubsection{Challenges}

The first challenge is that the theory above would not fit well with a theory of diachronic evolution of the slot structure of a slot (but see the footnote in 5.6 that briefly discusses whether we want to have a diachronic theory of IEs in a first place). Suppose that the slot ' $\mathrm{cn}_{0}[]$ ' has the slots ' $\mathrm{fn}_{0}[]$ ' and ' $\mathrm{n}_{0}[]$ ', and that the filler 'John Doe' has the slots 'fn $\mathrm{n}_{\mathrm{JD}}[]$ ' and ' $\ln _{\mathrm{JD}}[]$ '. As soon as 'John Doe' fills ' $\mathrm{cn}_{0}[]$ ', the slots 'f( $\mathrm{J}_{\mathrm{JD}}[]$ ' and ' $\ln _{\mathrm{JD}}[]$ ' appear in 'cno[]', and the slots fno[] and $\ln _{0}[]$ appear in 'John Doe'. We would like to have means to equate fno[] with fn $\mathrm{JD}_{\mathrm{D}}[]$, and $\ln _{0}[]$ with $\ln _{\mathrm{JD}}[]$ (or at least establish a mapping between them - see next subsection).

A second challenge pertains to supplementation. We have here accepted an axiom of weak supplementation involving both fillers and slots (AX11), similar to Bennett's. However, because our system accepts slots of slots, one might want to introduce axioms of supplementation purely at the level of slots, without any need of mediation by fillers (for an example of two-levels mereology, see the process specification language PSL, that endorses two mereologies, one at the level of activities through the relation "subactivity" and another one at the level of activity occurrences through the relation "subactivity_occurrence" [25]) . For this, we could first define a notion of overlap between slots as follows: s and t slot-overlap ("SO") just in case they share a slot $u$, one is a slot of the other, or they are identical (this latter mention is important, since a slot 
that does not have any slot does not share a slot with itself, but we want to state that any slot overlaps itself):

(DEF5) Slot-overlap SOtu:= ${ }_{\operatorname{def}}$ ISt \& ISu \& $[\exists v($ Svt \& Svu) $\vee$ Stu $\vee$ Sut V s=t $]$

This would enable to formulate an axiom of strong supplementation among slots:

(AX12) IS Strong Supplementation

ISt \& ISu \& $\mathrm{HSu} \rightarrow$

$[(\neg$ Sut $\& \mathrm{t} \neq \mathrm{u}) \rightarrow \exists \mathrm{v}($ Svu $\& \neg \mathrm{SOvt})]$

From this axiom, we could then easily deduce corresponding theorems of weak supplementation and extensionality on slots (using also AX6 to demonstrate the latter):

(TH12) IS Weak Supplementation $\quad$ ISu \& Stu $\rightarrow \exists v$ (Svu \& $\neg$ SOvt)

(TH13) IS Extensionality $\quad$ ISt \& ISu \& HSt \& HSu $\rightarrow[\mathrm{t}=\mathrm{u} \leftrightarrow \forall \mathrm{v}(\mathrm{Svt} \leftrightarrow \mathrm{Svu})]$

Ideally, we would want to use AX12 (IS Strong Supplementation) to prove IF Weak Supplementation as a theorem, instead of accepting it as an axiom (cf. AX11) as we did here. However, IS Extensionality is not compatible with our AX8. Indeed, if two slots are filled by the same filler that has slots, they have exactly the same slots by AX8; and by IS Extensionality (TH13), they would be identical. This would defeat the goal of this system that aims at enabling an IE to have a part twice (or more) over. To avoid this conclusion, one could relax AX8, for example by introducing a notion of "twin-slot".

\subsubsection{Twin-slots}

AX8 is equivalent to the conjunction of the two following axioms:

(AX8.1) Slots of a filled slot are slots of the filler Fxt \& Sut $\rightarrow$ Sux

(AX8.2) Slots of a filler are slots of the slot it fills Fxt \& Sux $\rightarrow$ Sut

To relax AX8, one could relax AX8.1, AX8.2, or both. However, if one (or both) of those axioms is abandoned, we may want to replace them by weaker axioms. For example, it seems sensible that because ' $\mathrm{cn}_{0}[]$ ' has two slots ' $\mathrm{fn}_{0}[]$ ' and ' $\mathrm{ln}_{0}[]$ ', a filler $\mathrm{x}$ of 'cno[]' should have two slots ' $\mathrm{fn}_{\mathrm{x}}\left[\mathrm{]}\right.$ ' and ' $\mathrm{n}_{\mathrm{x}}[\mathrm{]}$ '. To account for this and relax AX8.1, one could introduce the notion of "twin-slot" of a slot's slot. A minimal requirement for such twin-slot would be that if $x$ fills $t$ and $u_{t}$ is a slot of $t$, then there is a slot $u_{x}$ of $x$, called "twin-slot of $u_{t}$ in $x$ ", such that $u_{x}$ is filled by $y$ whenever $u_{t}$ is filled by $y$ :

(AX8.1') Twin-slot in a filler $\quad F x t \& \mathrm{Su}_{\mathrm{t}} \rightarrow \exists \mathrm{u}_{\mathrm{x}}\left[\mathrm{Su}_{\mathrm{x}} \mathrm{x} \& \forall \mathrm{y}\left(\mathrm{Fyu} \mathrm{u}_{\mathrm{t}} \leftrightarrow \mathrm{Fyu}_{\mathrm{x}}\right)\right]$

Using AX8.1', TH11 and AX4, we can show that a slot of $t$ and its twin slot in $x$ are filled by the same unique filler $\mathrm{y}$, proper part of $\mathrm{x}$ :

\section{(TH15) Filling of a twin-slot in a filler $\quad$ Fxt \& Sut $\rightarrow$ \\ $\exists \mathrm{u}_{\mathrm{x}} \exists$ !y $\left[\mathrm{Su}_{\mathrm{x}} \mathrm{x} \& \mathrm{Fyu}_{\mathrm{t}} \& \mathrm{Fyu}_{\mathrm{x}} \& \mathrm{PPyx}\right]$}

For example, if 'John Doe' fills 'cno[]', it has two slots 'fn $\mathrm{JD}_{\mathrm{J}}\left[\mathrm{c}\right.$ ' and ' $\mathrm{nn}_{\mathrm{JD}}[\mathrm{C}$ ' that are twin-slots of 'fno[]' and ' $\ln _{0}[]$ '. Both ' $\ln _{\mathrm{JD}}[]$ ' and ' $\mathrm{ln}_{0}[]$ ' are filled by 'Doe'; but if 'c $\mathrm{n}_{0}[]$ ' was instead filled by 'Jane Smith', then ' $\ln _{0}[]$ ' would be filled by 'Smith', whereas ' $\ln _{\mathrm{JD}}[]$ ' would still be filled by 'Doe' (and it would then not be a twin slot of ' $\ln _{0}[]$ ').

Similarly, one might also want to introduce the notion of twin-slot of a filler's slot. Future work should determine which of those axioms, and/or others, should be endorsed.

\subsection{Slot levels}

We may want to define a hierarchy of sublevels among slots. For example, a diagnostic report that would occupy a slot $\mathrm{s}_{0}$ could have the slots 'patient ${ }_{0}[]$ condition $_{0}[]$ doctor $_{0}[]$ ', where the slot 'patiento[]' is composed by the slots 'patient_fno[]' and 'patient_ln 0 []'. 
Note that according to AX7 Slot-of transitivity, 'patient_fno[]' and 'patient_ $\ln _{0}[]$ ' are also slots of $s_{0}$. But we might want to state that the slots 'patient 0[] ', 'condition 0 []' and 'doctoro[]' are first-sublevel (or direct) slots of so, whereas the slots 'patient_fno[]' and 'patient_lno[]' are second-sublevel slots of so. We can define the various sublevels as follows (see [26] for an axiomatization):

- A $1^{\text {st }}$-level slot of $\mathrm{s}$ is a slot of $\mathrm{s}$ that is not a slot of a slot of $\mathrm{s}$.

- A $2^{\text {nd }-l e v e l ~ s l o t ~ o f ~} s$ is a slot of a slot of $s$ that is not a slot of a slot of a slot of s (etc.)

Note that in our axiomatization, there is no axiom that forces the existence of $1^{\text {st }}$-level slots, $2^{\text {nd }}$-level slots, etc. That is, there is no discreteness axiom that rules out the existence of a dense set of sublevels. Such axioms might be added in future work.

\subsection{Mereological sum}

We may want to formally introduce the mereological sum of several fillers of several slots. For example, elaborating on AX10, we may want to state that if all slots of a slot s are filled, the filler that fills $\mathrm{s}$ is the mereological sum of those fillers.

More innovatively, we may want to introduce an entity that would be composed by a filler and the slot it fills, such as the slot 'patient[]o' and its filler 'John Doe'. This would indeed have consequences for aboutness (see section 5.4). A possible way might be to represent this as a mereological sum of the filler and its slot. Such an account could be compared to Koslicki's theory of the composition of material objects [27], which holds that objects have two proper parts: material parts and formal parts. As her analysis goes, for instance, Michelangelo's statue David is composed of an amount of marble (material part) and, say, the "David-wise structure" (formal part). Indeed, Koslicki states that a general notion of structure can be characterized as an entity that offers available "positions or places" (reminiscent of our "slots"). A mereological sum of a filler and its slot that would be completed by some kind of arrangement among subslots would thus seem to be very much in Koslicki's spirit.

\subsection{Aboutness}

We will not propose a formal theory of aboutness, but only give a few pointers of how we could extend IAO's theory of aboutness with an aboutness of ISs. We will consider here that an informational entity can be about several kinds of entities, such as a particular, a class, or a state of affairs [14]. This is relatively classical at the level of fillers. For example, in $\operatorname{diag}_{0}$, 'John Doe' is about the particular human John Doe and 'Flu' is about the class Flu. However, we suggest that slots can also be about a variety of things. For example, 'patiento[]' would be about the class Patient and 'conditiono[]' would be about the class Medical condition. Then, if we accept the mereological sum of a slot and its filler as explained earlier, the sum of 'John Doe' and the slot it occupies 'patient ${ }_{0}[]$ ' might be about the relation of instantiation of the class Patient by the particular John Doe. Note however that not all slots are about something - consider e.g. the slot ' $2^{\text {nd }}$ lettero[]'. Such considerations should be integrated into a full theory of aboutness of ICE - something that still needs to be developed in IAO.

\subsection{Refusing supplementation}

There might be reasons to refuse AX11 (IF Weak Supplementation) in our mereological system. Indeed, we might want to accept to have PPxy while y having as 
only slots the slot filled by $\mathrm{x}$ and the slots of $\mathrm{x}$. For example, suppose that Mr. J's last name is composed by only one letter, ' $\mathrm{J}$ '. He needs to fill some administrative form that has the slot 'last name $e_{0}[]$ '. He fills it with ' $\mathrm{J}$ '. The slot 'last name $\mathrm{e}_{0}[]$ ' is filled with this filler 'J'Name, that is about Mr. J. And this filler has one unique slot 'first letterJ[]', that is filled with the filler ' $\mathrm{J}$ ' Letter, which is a letter that is about nothing. That is, Mr. J fills two slots by drawing the same sign. And by AX8, 'last name ${ }_{0}[]$ ' would have a unique slot 'first letter []]'.

However, following a suggestion by Masolo and Vieu [2], one might instead introduce a relation of composition between a word and the chain of characters it is made of, such that this relation would not be identical to parthood (for more on composition, see [28]). In such a case, 'J'Name would be constituted by the character 'J'Letter, but would not have it as part, and thus it would not be a counter-example to supplementation axioms. Moreover, such an approach could explain the change in aboutness when we move from letters to words.

\subsection{The diachronic identity of documents and creation of ISs}

The diachronic identity of documents is a topic that has been little studied. To illustrate its complexity, suppose that I start working on a homework. In front of me, I have a blank sheet of paper. At $t_{1}$, I decide that this paper will be the physical carrier of my homework: I decide that I will write my name on the top left, the date on the top right, the body of my text on the paper, divided in three parts. At $t_{2}$, I have written my name on the top left. and the date on the top right. At $t_{3}$, I have written the three parts of the body of my text.

It would be desirable to have a theory of identity according to which it is the same homework that evolves while I am filling it; that is, that there exists a unique homework IE at $t_{1}, t_{2}$ and $t_{3}$. The theory we developed earlier is compatible with such diachronic identity considerations: the homework remains the same document from $t_{1}$ to $t_{3}$, although some new parts (new IFs) appear ${ }^{3}$.

Interestingly, several relevant ISs have arguably already appeared at $\mathrm{t}_{1}$. Of course, we do not claim that something physically changed in the composition of the sheet of paper when I made this decision at $t_{1}$ to write my homework on this sheet of paper. The fact that it changed while not changing in physical structure (thus undergoing a Cambridge change [30]) only emphasizes the cognitive and social nature of ISs (something that is also true for IFs, as they depend on the existence of a semiotic system): it is my cognitive act (maybe mirrored in other cognitive agents) to structure my document with a name, a date, and a body of text in three parts that created those ISs.

Similarly, a database, a patient chart or a drug distribution report can remain identical while changing in content. Note that the number and nature of the ISs of an entity can change in two ways. First, trivially, IFs will themselves have ISs, which will, by slot inheritance, be ISs of the overall documents; therefore, new slots appear (consider again the "Jean-Marc" example in 4.1.4). Second, the ISs of a document can change depending on how agents change another part of the document. Consider for example a response sheet to a poll, where a positive answer to "Are you a national of another country than Canada?" will bring the IS to be filled by the nationality of the respondent.

\footnotetext{
${ }^{3}$ Note that this raises an interesting difficulty: if I make two copies of the homework at an earlier stage of development and then both evolve in different ways, it seems that they would remain identical despite evolving differently, which is counter-intuitive. This kind of problem has been studied by Parfit in the case of personal identity [29]. One possible solution, in a Parfitian spirit, would then be to abandon the notion of diachronic identity for informational entities.
} 


\subsection{Feature structures}

As mentioned earlier, this system has analogies with the theory of feature structures [31]. The latter theory also introduces slots, that can be recursively filled by feature structures. A difference is that the root element in this theory is a feature structure, whereas in our theory, the root element is a slot (that is, a filler always fills a slot). Another difference is that feature structures are naturally interpretable as types, whereas fillers are first and foremost tokens (particulars) in our theory (although we can introduce classes of fillers). Finally, classical mereological constraints such as supplementation do not appear in the theory of feature structures.

\section{Conclusion}

We have introduced an axiomatic system for the mereology of informational entities using the notions of information slots and information fillers. Inspired by Bennett, this system is different in several important respects, in particular in having free-floating slots, slots of slots and empty slots.

This work has been extended by a companion paper investigating the notions of adequate vs. inadequate filling of a slot, as well as levels of slots, with a focus on clinical documents [26]. Important future investigations will include considering to relax AX8, introducing slot supplementation axioms and mereological sums, as well as analyzing the aboutness of slots and fillers. One could also investigate whether unfilled slots could be replaced or complemented by slots filled by an empty filler ' $\emptyset$ ': for example, a blank exam template might be unfilled before the exam sheets are distributed, whereas if I return my exam sheet empty, one could consider that I have filled the slots of this document with the empty filler. Finally, one should analyze the various forms in which IFs and ISs can be concretized, in particular when a message is conveyed as a process (e.g. spoken language or Morse code). This could strengthen the basis of ontologies of informational entities such as IAO, the ontology of document acts [32], and domain ontologies founded on them, such as the Prescription of Drugs Ontology (PDRO) [33] or the LABoratory Ontology (LABO) [34].

\section{Acknowledgements}

We thank four reviewers as well as Benoît Fraikin, Rosalie Plantefève and the audience at a seminar in Sherbrooke university in July 2020 for their insightful comments. FT acknowledges financial support by the SPOR Canadian Data Platform (CIHR).

\section{References}

[1] Barton A, Fabry P, Ethier J-F. A classification of instructions in drug prescriptions and pharmacist documents. Proc 10th Int Conf Biomed Ontol ICBO 2019. Buffalo, New York, USA; accepted. p. 1-7.

[2] Masolo C, Vieu L. Graph-Based Approaches to Structural Universals and Complex States of Affairs. In: Borgo S, Hitzler P, Kutz O, editors. Form Ontol Inf Syst Proc 10th Int Conf FOIS 2018. IOS Press; 2018. p. 69-82.

[3] Arp R, Smith B, Spear AD. Building Ontologies with Basic Formal Ontology. The MIT Press; 2015.

[4] Bennett K. Having a part twice over. Australas J Philos. 2013;91:83-103. 
[5] Simons P. Parts: A study in ontology. Oxford: Clarendon Press; 1987.

[6] Casati R, Varzi AC. Parts and places: The structures of spatial representation. Cambridge, MA: Mit Press; 1999.

[7] Masolo C, Borgo S, Gangemi A, et al. The WonderWeb library of foundational ontologies and the DOLCE ontology. WonderWeb (EU IST project 2001-33052) deliverable D18. LOA-ISTC-CNR; 2003.

[8] Cotnoir AJ. Strange parts: The metaphysics of non-classical mereologies. Philos Compass. 2013;8:834 845.

[9] Tsai H. Decidability of mereological theories. Log Log Philos. 2009;18:45-63.

[10] Effingham N, Robson J. A mereological challenge to endurantism. Australas J Philos. 2007;85:633-640.

[11] Cancedda F. Functional requirements for authority data: a conceptual model: Final report, December 2008. Boll AIB 1992-2011. 2010;50:131-132.

[12] Peroni S, Palmirani M, Vitali F. UNDO: the United Nations system document ontology. Int Semantic Web Conf. Springer; 2017. p. 175-183.

[13] Bountouri L, Gergatsoulis M. The semantic mapping of archival metadata to the CIDOC CRM ontology. J Arch Organ. 2011;9:174-207.

[14] Smith B, Ceusters W. Aboutness: Towards foundations for the information artifact ontology. Proc 6th Int Conf Biomed Ontol. Lisbon, Portugal: CEUR Workshop Proceedings; 2015. p. 1-5.

[15] Rudnicki R. An Overview of the Common Core Ontologies [Internet]. Buffalo: CUBRC, Inc.; 2019. Available from: https:/www.nist.gov/system/files/documents/2019/05/30/nist-ai-rfi-cubrc_inc_004.pdf.

[16] Garbacz P. A formal ontology of texts. In: Ferrario R, Kuhn W, editors. Form Ontol Inf Syst Proc 9th Int Conf FOIS 2016 [Internet]. Amsterdam: IOS Press; 2016. p. 345-358. Available from: http://ebooks.iospress.com/volumearticle/44240.

[17] Masolo C, Vieu L, Bottazzi E, et al. Social Roles and their Descriptions. In: Dubois D, Welty C, editors. Proc 9th Int Conf Princ Knowl Represent Reason KR 2004. Menlo Park, CA: AAAI Press; 2004. p. 267 277.

[18] Arora N, Westenthaler R, Behrendt W, et al. Information object design pattern for modeling domain specific knowledge. 1st ECOOP Workshop Domain-Specif Program Dev. 2006.

[19] Hawke P. Theories of aboutness. Australas J Philos. 2018;96:697-723.

[20] Bateman JA. Ontology, Language, Meaning: Semiotic Steps Beyond the Information Artifact. Ontol Makes Sense Essays Honor Nicola Guarino. Amsterdam: IOS Press; 2019. p. 119-135.

[21] Tarski A. The concept of truth in formalized languages. Log Semant Metamathematics. 1956;2:7.

[22] Corcoran J, Frank W, Maloney M. String theory. J Symb Log. 1974;625-637.

[23] Galton A. On generically dependent entities. Appl Ontol. 2014;9:129-153.

[24] Sattig T. Part, slot, ground: foundations for neo-Aristotelian mereology. Synthese. 2019;1-15.

[25] Grüninger M. Using the PSL ontology. Handb Ontol. Springer; 2009. p. 423-443.

[26] Barton A, Toyoshima F, Ethier J-F. Clinical documents and their parts. Proc 11th Int Conf Biomed Ontol ICBO 2020. accepted.

[27] Koslicki K. The structure of objects. Oxford University Press on Demand; 2008.

[28] Masolo C, Vieu L, Ferrario R, et al. Collective, composites and pluralities. In: Broadaric B, Neuhaus F, editors. Form Ontol Inf Syst Proc 11th Int Conf FOIS 2020. Bolzano, Italy: IOS Press; accepted.

[29] Parfit D. Reasons and persons. OUP Oxford; 1984.

[30] Mortensen C. Change and Inconsistency. In: Zalta EN, editor. Stanf Encycl Philos [Internet]. Spring 2020. Metaphysics Research Lab, Stanford University; 2020. Available from: https://plato.stanford.edu/archives/spr2020/entries/change/.

[31] Carpenter B. The logic of typed feature structures: with applications to unification grammars, logic programs and constraint resolution. Cambridge University Press; 2005.

[32] Brochhausen M, Almeida MB, Slaughter L. Towards a formal representation of document acts and resulting legal entities. Johanssonian Investig Essays Honour Ingvar Johansson His Seventieth Birthd. Walter de Gruyter; 2013.

[33] Ethier J-F, Barton A, Taseen R. An ontological analysis of drug prescriptions. Appl Ontol. 2018;13:273294.

[34] Barton A, Fabry P, Lavoie L, et al. LABO: An ontology for laboratory test prescription and reporting. In: Barton A, Seppälä S, Porello D, editors. Proc Jt Ontol Workshop 2019 JOWO 2019. Graz, Autriche; 2019. p. 1-9. 